\title{
Proactive measurement of infliximab drug levels in children with Crohn's disease
}

\author{
Kathleen Hollanda, William E. Bennett ${ }^{b}$, James E. Slavenc, John Collier ${ }^{d}$, Gail Waltze, Marian Pfefferkorn ${ }^{f}$ \\ Indiana University School of Medicine, Indianapolis, IN; University of Utah School of Medicine, Salt Lake City, \\ Utah, USA
}

\begin{abstract}
Background Proactively monitoring infliximab levels is an emerging area of interest in pediatric Crohn's disease. There are only limited data on therapeutic drug monitoring for children with Crohn's disease. The goal of our study was to determine the utility of therapeutic drug monitoring in achieving clinical remission in a cohort of pediatric Crohn's disease patients receiving infliximab.

Methods This prospective single-center study enrolled 37 patients with Crohn's disease at the start of infliximab infusions and monitored trough levels at 6-month intervals for 18 months. Each participant was matched to a historic control for the modified pediatric Crohn's disease activity index (mPCDAI) at baseline, age and sex. The primary outcome was an mPCDAI score of $\leq 7.5$ at 6, 12 and 18 months. A multivariate logistic regression analysis was performed.

Results Data were available for all 37 cases at 6 and 12 months and for 34 cases at 18 months. Demographics and disease characteristics were similar between groups. All 34 cases demonstrated clinical remission at 18 months $(100 \%$ vs. $88 \%, \mathrm{P}=0.114)$. Univariate and multivariate analyses did not show statistical significance. Dose intensification was seen more often in the cases at 18 months.

Conclusion All of our moderate-to-severe pediatric Crohn's disease patients who received prospective therapeutic drug monitoring of infliximab were in clinical remission at follow up, but this was not statistically significantly different from the $88 \%$ clinical remission rate of the control group.
\end{abstract}

Keywords Therapeutic drug monitoring, Crohn's disease, infliximab

Ann Gastroenterol 2021; 34 XX: 1-7

\section{Introduction}

The management of pediatric Crohn's disease (CD) has changed significantly over the last decade as more targeted monoclonal antibodies have been developed. With the

${ }^{\mathrm{a}} \mathrm{GI}$ Consultants, Reno, NV, USA (Kathleen Holland); ${ }^{\mathrm{b}}$ Department of Pediatrics, Indiana University School of Medicine, Indianapolis, IN, USA (William E. Bennett); ' Department of Biostatistics, Indiana University School of Medicine, Indianapolis, IN, USA (James E. Slaven); ${ }^{\mathrm{D}}$ Department of Pediatrics, University of Utah School of Medicine, Salt Lake City, Utah, USA (John Collier); ${ }^{e}$ Department of Pediatrics, Riley Hospital for Children, Indianapolis, IN, USA (Gail Waltz); fDepartment of Pediatrics, Indiana University School of Medicine, USA (Marian Pfefferkorn)

Conflict of Interest: None

Correspondence to: Marian Pfefferkorn M.D., 705 Riley Hospital Drive \#4210, Indianapolis, IN 46202, USA, e-mail: mdelrosa@iu.edu

Received 13 April 2021; accepted 30 August 2021;

published online 10 November 2021

DOI: https://doi.org/10.20524/aog.2021.0676 increased use of anti-tumor necrosis factor (TNF) agents such as infliximab (IFX), the question remains as to how best to monitor efficacy. A substantial amount of adult and some pediatric data have demonstrated the effectiveness of IFX in mucosal healing and maintenance of clinical remission when also used in conjunction with an immunomodulator [1-7]. Patients at risk for moderate-to-severe disease may benefit from a biologic given early on [3,4,8-10].

A newer focus when using anti-TNFs such as IFX is how best to monitor them, with the goal of using the most efficacious dose to achieve remission. The European Society for Paediatric Gastroenterology, Hepatology and Nutrition (ESPGHAN) currently supports a $5 \mathrm{mg} / \mathrm{kg}$ dose for all 3 IFX infusions during the induction phase of treatment [11]. Treatment escalation, by either increasing drug dosage or decreasing the interval between infusions, may be helpful for maintaining clinical remission [12]. Therapeutic drug monitoring (TDM) has gained favor, simply because it allows practitioners to tailor treatment to each individual [13]. Most of what we know about TDM and IFX is from retrospective or observational adult studies. The Trough Concentration Adapted Infliximab Treatment (TAXIT) trial 
evaluated the effectiveness of TDM in adult patients with CD and ulcerative colitis (UC) on IFX maintenance therapy. Two groups were randomized to dose escalation, based either on drug levels or on clinical symptoms and C-reactive protein (CRP). The primary endpoints were improved clinical and biological remission one year after dose optimization. Unfortunately, there was no statistical difference between the 2 groups as regards the primary endpoints, but there was a significant difference in the rates of disease relapse [12].

Studies on proactive TDM in children are scarce, although some evidence suggests that monitoring levels during induction may be beneficial, as well as aiming for even higher trough levels at the start of maintenance infusions. A prospective observational study in children aimed to understand how IFX trough levels and high IFX antibody levels may alter clinical remission at week 54. IFX trough levels of $>7 \mu \mathrm{g} / \mathrm{mL}$ at week 14 had $100 \%$ positive predictive value (PPV) in predicting persistent remission over the first year [14]. Persistent remission was defined as clinical remission evidenced by a pediatric $C D$ activity index (PCDAI) level of $<10$ and no dose escalation or intensification [14]. Another pediatric prospective observational study assessed how best to monitor IFX trough levels during induction and what levels to aim for in regard to clinical and biological remission (CRP and fecal calprotectin) at the start of maintenance therapy. Results showed IFX trough levels of $\geq 29$ prior to infusion 2 and $\geq 18 \mu \mathrm{g} / \mathrm{mL}$ prior to infusion 3 resulted in improved PCDAI scores and normalization of fecal calprotectin levels and CRP [15].

Monitoring for clinical remission with scoring systems such as the PCDAI is less invasive than endoscopic surveillance and has been the mainstay for monitoring disease activity in children. A multicenter prospective study in 2005 aimed to identify cutoff values for PCDAI to classify children with CD as mild, moderate or severe. A value $\leq 10$ is indicative of remission, while a value $\geq 30$ shows moderate/severe disease [16]. Given that predictors, including height, velocity and perianal disease assessment, are difficult to obtain at each visit, the modified PCDAI (mPCDAI) scoring systems have been validated in the literature. A mPCDAI $\leq 7.5$ can be used to determine clinical remission [17].

To generate additional data about TDM in children with $\mathrm{CD}$, we prospectively monitored IFX trough levels over the course of 18 months to determine improved clinical remission rates (defined as mPCDAI scores $\leq 7.5$ ). We then compared remission rates between this cohort and a historic cohort of matched controls. We hypothesized that TDM would increase the rate of clinical remission in the monitored cohort at 6,12 and 18 months.

\section{Patients and methods}

Children between the ages of 5-20 years with established CD and starting IFX induction were prospectively enrolled in the study between 2014-2019 at the Riley Hospital for Children at Indiana University Health, in Indianapolis, IN.
Only 2 children were older than 18 years over the course of the study. These children are followed by one of the 16 pediatric gastroenterologists in the practice. Trough levels were collected prior to the fourth infusion ( $1^{\text {st }}$ maintenance infusion) and with every infusion until a level of $\geq 4 \mu \mathrm{g} / \mathrm{mL}$ was attained, and then monitored every 6 months for 18 months. IFX and drug antibody levels were measured at the Prometheus Laboratories, Inc. (San Diego, CA, USA) using the homogeneous mobility shift assay. A level $\geq 4 \mu \mathrm{g} / \mathrm{mL}$ was chosen as our therapeutic cutoff, as this has been shown in previous prospective pediatric studies to have a PPV of $76 \%$ in predicting remission at week 54 [14].

The mPCDAI score was calculated every 6 months and recorded along with trough levels and demographic information. Additionally, we assessed the following disease characteristics for each participant: use of steroids within 1 month of starting IFX infusions; use of immunomodulators at any point during the 18 months of IFX infusions; presence of perianal/fistulizing disease; and any additional procedures (stricture dilations, intestinal resections, or intra-abdominal abscess drainage). Demographics included age at diagnosis, sex, race, and insurance type. We anticipated missing data and chose to perform a complete case analysis in which we only analyzed patients able to complete at least 12 months of the study.

Each of the cases were matched with historic controls at our institution in a 1:1 fashion. These controls were collected from the ImproveCareNow (ICN) registry between 2014 and 2018. They were first matched for mPCDAI score at the start of IFX infusions, then by age and finally by sex. Unfortunately, there was no protocol for TDM at our center during this period, and thus the majority of patients are missing IFX levels.

Prior to enrolment, the study was approved by the Indiana University Institutional Review Board with expedited status.

\section{Statistical analysis}

Bivariate matched analysis using McNamar's tests were performed to account for the matched/paired data and to determine the proportion of patients with mPCDAI $\leq 7.5$ at 6,12 and 18 months. McNamar's tests were also used to determine if there were significant bivariate associations between case-control and the main outcome variables of mPCDAI categories at each follow-up time point (mPCDAI $\leq 7.5$ at 6 months, 12 months, and 18 months), as well as for other demographic variables (insurance, mPCDAI at the start of IFX infusions, use of immunomodulators, and use of steroids within 1 month of starting IFX). Clinical remission was considered when the mPCDAI was $\leq 7.5$ at each of the time points. Unadjusted logistic regression models were then constructed, using the Newton-Raphson Ridge Optimization algorithm to model the matching schema. Adjusted models, including the other demographic/disease variables (Medicaid, immunomodulator and steroids within 1 month of starting IFX) and mPCDAI category at baseline were included in the final model. Disease characteristics were chosen for the model based on the possibility that 
these medications could help control disease activity, which might affect baseline mPCDAI. Medicaid was chosen as a covariate, given previous evidence that children living in lower-income families and with chronic medical conditions

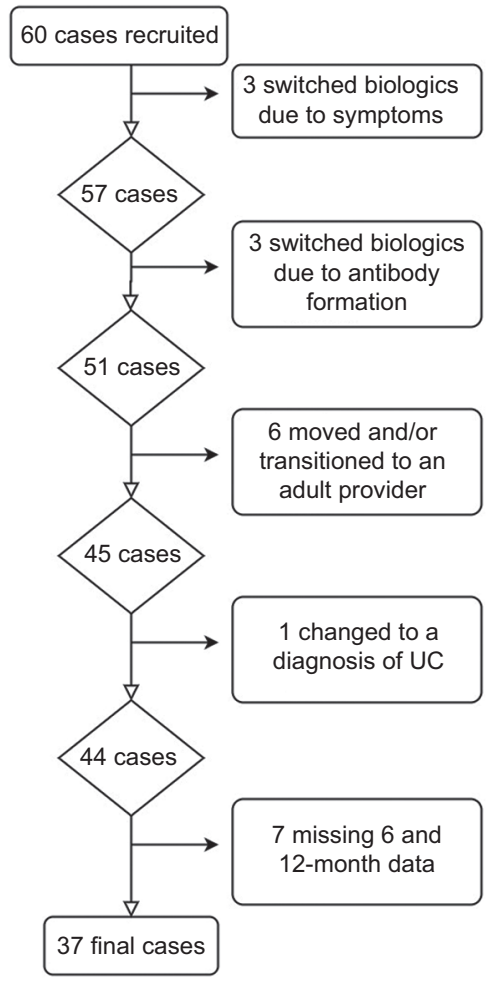

Figure 1 Flow diagram for final cases $U C$, ulcerative colitis tend to suffer worse health outcomes, including quality of life scores [18]. All analytic assumptions were verified, and analyses were performed using SAS v9.4 (SAS Institute, Cary, NC).

\section{Results}

An initial 60 participants were enrolled in the study, with 23 being excluded early on for various reasons, including: 1) switching to different biologics; 2) moving out of state; 3 ) transitioning to an adult provider; and 4) change of diagnosis to UC (Fig. 1). A final number of 37 cases and 37 controls were included in the study. Similar demographics were observed between the cases and controls (Table 1). Most participants were Caucasian and on private insurance. The average age at diagnosis for cases was 16.24 years (standard deviation [SD] 2.77) and 16.84 years (SD 2.65) for controls. In regard to disease characteristics, most of the cases and controls were not on steroids within 1 month of starting IFX, only about $20 \%(n=7)$ had fistulizing/perianal disease, and a minority had had gastrointestinal surgeries $(n=3)$. Greater than $50 \%$ $(n=20)$ of both cases and controls were on dual therapy with an immunomodulator and IFX during the 18-month course of this study (Table 1).

\section{IFX trough levels}

We analyzed the average IFX trough levels $(\mu \mathrm{g} / \mathrm{mL})$ prior to the fourth infusion (first maintenance) and at 6 ,

Table 1 Case and control demographics and disease characteristics of children with Crohn's disease on IFX (2015-2018)

\begin{tabular}{|c|c|c|c|c|c|}
\hline \multirow[t]{3}{*}{ Characteristics } & \multirow{2}{*}{\multicolumn{2}{|c|}{$\begin{array}{l}\text { Cases } \\
(\mathrm{n}=37)\end{array}$}} & \multirow{2}{*}{\multicolumn{2}{|c|}{$\begin{array}{l}\text { Controls } \\
(n=37)\end{array}$}} & \multirow[t]{3}{*}{ P-value } \\
\hline & & & & & \\
\hline & $\mathrm{n}$ & $\%$ & $\mathrm{n}$ & $\%$ & \\
\hline Male & 24 & $65 \%$ & 24 & $65 \%$ & $>0.99$ \\
\hline Caucasian & 32 & $86 \%$ & 31 & $84 \%$ & 0.763 \\
\hline Medicaid & 6 & $16 \%$ & 3 & $8 \%$ & 0.317 \\
\hline Immunomodulator $^{\mathrm{a}}$ & 20 & $54 \%$ & 24 & $65 \%$ & 0.346 \\
\hline Steroids ${ }^{b}$ & 11 & $30 \%$ & 9 & $24 \%$ & 0.564 \\
\hline GI surgery ${ }^{c}$ & 3 & $8 \%$ & 3 & $8 \%$ & $>0.99$ \\
\hline Fistulizing/perianal disease $^{d}$ & 7 & $19 \%$ & 10 & $27 \%$ & 0.366 \\
\hline Average age & 16.24 & (SD 2.77) & 16.84 & (SD 2.65) & 0.110 \\
\hline Average mPCDAI at start of IFX & 19 & (SD15) & 20 & (SD13) & 0.760 \\
\hline \multicolumn{6}{|c|}{ Values are frequencies (percentages) with P-values from McNemar's tests. Means calculated using paired $t$-test } \\
\hline \multicolumn{6}{|c|}{ a Use of an immunomodulator over 18 months } \\
\hline \multicolumn{6}{|c|}{ bUse of steroids within 1 month of starting infliximab } \\
\hline \multicolumn{6}{|c|}{ 'History of GI surgery (resections, abscess drainage) } \\
\hline${ }^{\mathrm{d}}$ History of fistulizing or perianal dise & & & & & \\
\hline
\end{tabular}


12 , and 18 months after a therapeutic level of $\geq 4 \mu \mathrm{g} / \mathrm{mL}$ was obtained (Fig. 2). Based on these data, we observed a wide variability in trough concentrations at the start of maintenance, with the majority reaching the target goal of $\geq 4 \mu \mathrm{g} / \mathrm{mL}$. The trough drug levels gradually increased over the 18 months. The average concentration increased from a median score of $8.5 \mu \mathrm{g} / \mathrm{mL}$ at the start of maintenance to 11.6 $\mu \mathrm{g} / \mathrm{mL}$ at 6 months, $13.3 \mu \mathrm{g} / \mathrm{mL}$ at 12 months, and $12.3 \mu \mathrm{g} /$ $\mathrm{mL}$ at 18 months. Eighty-one percent of the cases reached a trough level of at least $4 \mu \mathrm{g} / \mathrm{mL}$ at the start of maintenance. Unfortunately, we did not have these data available for the controls. It is also important to note that 3 of 37 cases (8\%) developed anti-drug antibodies (ADA) that were all less than $10 \mathrm{U} / \mathrm{mL}$ throughout the 18 months course, none of which prompted a medication change. Similar data were not available for the controls.

\section{Dose optimization}

We analyzed an increased frequency of infusions ( $<8$-week intervals) and higher doses ( $>5 \mathrm{mg} / \mathrm{kg}$ ) of IFX given over the 18 months, comparing cases and controls (Supplementary Fig. 1,2). Cases were far more likely to receive higher than $5 \mathrm{mg} / \mathrm{kg}$ doses of IFX and more frequent infusions ( $<8$-week intervals) over the 18 months. Higher dose IFX concentrations were statistically significant at each of the time intervals between cases and controls (Supplementary Fig. 1) as well as $<8$-week IFX infusion intervals at 12 months (Supplementary Fig. 2). Among the 28 cases that required dose intensification during the 18-month study, 50\% (14 patients) required intensification based on symptoms and biological markers, including CRP, erythrocyte sedimentation rate (ESR) and/or fecal calprotectin, while only $25 \%$ (7 patients) were escalated secondary to low IFX levels and another 7\% (2 patients) secondary to antibody levels (all $<10 \mathrm{U} / \mathrm{mL}$ ). The reasons for dose escalation in the final $18 \%$ (5 patients) were not clear from the documentation.

\section{mPCDAl results at 6,12 and 18 months}

Our primary outcome of interest was mPCDAI $\leq 7.5$ (clinical remission) at 6,12 and 18 months. The average mPCDAI at the start of infusions was 19 for cases and 20 for controls. Fig. 3 compares the percentages of cases and controls with $\mathrm{mPCDAI} \leq 7.5$ at 6,12 and 18 months. A larger percentage of cases at the start of IFX maintenance infusions already had mPCDAIs $\leq 7.5$ compared to controls $(29.7 \%$ vs. $16.2 \%$, $\mathrm{P}=0.268$ ). Patients in both the case and control groups similarly displayed improved mPCDAIs at 6 and 12 months. Eightythree percent of controls and $78 \%$ of cases achieved clinical remission (mPCDAI $\leq 7.5)$ at 6 months, and $97 \%$ controls vs. $81 \%$ of cases achieved clinical remission at 12 months $(\mathrm{P}=0.767$ and $\mathrm{P}=0.055$, respectively). At 18 months, all 34 cases were in clinical remission (mPCDAI $\leq 7.5)$ compared to 30 controls (100\% vs. $88 \%, \mathrm{P}=0.114$ ) (Fig. 3 ).

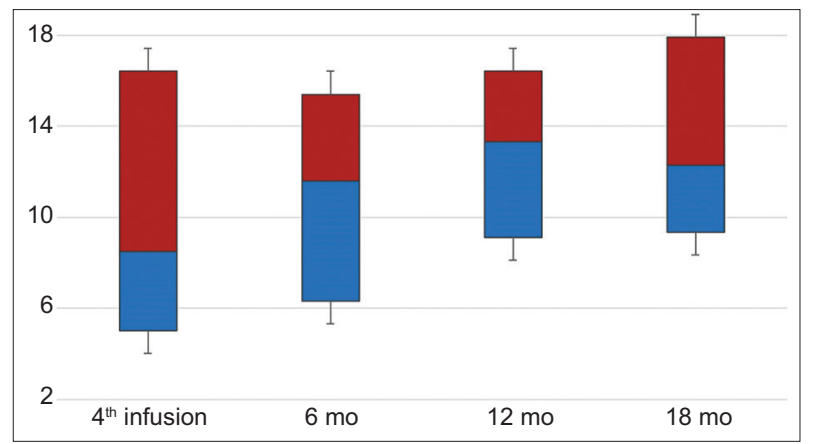

Figure 2 Average IFX trough concentrations $(\mu \mathrm{g} / \mathrm{ml})$ for cases at $4^{\text {th }}$ infusion, 6, 12 and 18 months

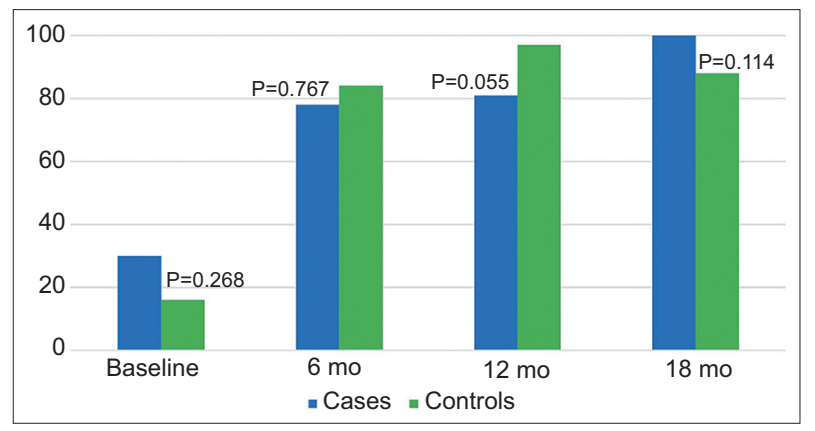

Figure 3 Percentage of mPCDAI $<7.5$ at baseline, b, 12 and 18 months Baseline is at the start of infliximab induction infusions

Values are frequencies (percentages) with P-values from McNemar's tests

When analyzing the groups at each time point across 18 months, we noted some important differences. Among the 34 cases and controls who had data points at 18 months, $25(73 \%)$ of the cases and $27(79 \%)$ controls remained in remission at all 3 time points $(\mathrm{P}=0.888)$.

\section{Univariate and multivariate regression}

Using bivariate analysis and case-control matching, we analyzed mPCDAI $\leq 7.5$ at 6,12 and 18 months between cases and controls. The results are shown in Table 2. Based on these results, we found no statistical difference between the cases and controls in regard to clinical remission over the 18-month period, other than the univariate results at 12 months, which showed better mPCDAI for controls vs. cases $(P=0.055)$. Similar results were discovered in the conditional multivariate regression model, adjusting for steroid exposure within a month of starting IFX, Medicaid insurance and use of an immunomodulator during the study (Table 2). No statistically significant difference was noted between the cases and controls when adjusting for the covariates mentioned above. In addition, the multivariate analyses did not include the Newton-Raphson Ridge Optimization algorithm, as the model would not converge. The results for treatment, however, remained similar in effect 
Table 2 Comparing odds ratios for modified pediatric Crohn's disease activity index $\leq 7.5$ at 6, 12 and 18 months in children with Crohn's disease on infliximab

\begin{tabular}{|c|c|c|c|c|c|c|}
\hline \multirow[t]{2}{*}{ Time points } & \multicolumn{3}{|c|}{ Univariate } & \multicolumn{3}{|c|}{ Multivariate } \\
\hline & OR & $95 \%$ CI & P-value & OR & $95 \%$ CI & P-value \\
\hline 6 months & 0.60 & $0.14-2.51$ & 0.484 & 0.80 & $0.24-2.72$ & 0.725 \\
\hline 12 months & 0.12 & $0.01-1.02$ & 0.052 & 0.12 & $0.01-1.05$ & 0.055 \\
\hline 18 months & $>99$ & $0.01->99$ & 0.946 & $>99$ & $0.01->99$ & 0.939 \\
\hline
\end{tabular}

Multivariate analysis controlled for Medicaid, immunomodulator and steroids in the final model

OR, odds ratio; CI, confidence interval

size between these multivariable models and the matched bivariate analyses.

\section{Discussion}

We performed a cohort study using historic controls of TDM in children with established CD receiving IFX infusions. Our results showed $100 \%$ clinical remission rates at 18 months in cases compared to $88 \%$ in controls, although the difference did not achieve statistical significance when using this strategy. While we did see improvements in clinical remission at 6 and 12 months among the cases, controls showed higher rates across these 2 time points.

TDM offers practitioners a useful way to monitor effectiveness and tailor drug therapy to each individual child with $\mathrm{CD}$ in hopes of maintaining drug efficacy and durability. The American Gastroenterological Association (AGA), however, does not recommend proactive anti-TNF drug monitoring yet, because of the inconclusive data thus far. The AGA currently recommends reactive drug monitoring in adults on anti-TNF agents [19]. Recent data in children with inflammatory bowel disease (IBD) on IFX showed that children may need higher trough levels during induction, and a trough level of at least $7 \mu \mathrm{g} / \mathrm{mL}$ at week 14 may be needed to preserve clinical remission at 1 year $[16,17]$. Our data indicate that a lower target level of at least $4 \mu \mathrm{g}$ / $\mathrm{mL}$ shows similar remission rates. No studies to date have examined whether or not proactively monitoring IFX trough levels in children with CD improves clinical remission scores (mPCDAI).

In our study, all 34 patients with complete data at 18 months were in clinical remission, based on an $\mathrm{mPCDAI} \leq 7.5$, compared to 34 of 37 controls. While there was a numerical difference in remission between the 2 groups favoring the cases, this did not reach statistical significance. We attribute this interesting finding to the likelihood that all our moderate-to-severe CD patients are closely monitored in the outpatient setting. The astute gastroenterologist is probably able to adjust the IFX dose based on clinical cues observed and the laboratory monitoring our patients receive during each IFX infusion. Minar et al have shown that abnormal ESR and albumin were significantly associated with subtherapeutic IFX concentrations [20].
With our prospective study, we did establish that cases were more likely to receive dose intensification strategies compared to controls. Interestingly, we did note that, in the $50 \%$ of cases who required dose intensification, this was secondary to clinical symptoms/biological markers and not secondary to IFX levels. One potential reason for this is that patients are frequently seen at the time of their infusions prior to IFX levels coming back. Providers probably come to a decision on IFX dose intensification following review of patient symptoms and clinical data at that clinic visit.

A 2019 retrospective study by Bauman et al set out to assess whether dose escalation or decreased frequency of IFX infusions in children with IBD played a role in predicting target trough levels $(\geq 5 \mu \mathrm{g} / \mathrm{mL})$. Shortening infusions to every 4 weeks at a dose of $5 \mathrm{mg} / \mathrm{kg}$ increased the percentage of patients meeting goal trough level from $24-84 \%$. When the dose was doubled to $10 \mathrm{mg} / \mathrm{kg}$, but with infusion intervals kept at every 8 weeks, goal trough levels were only obtained in 56\% of children. They also validated the total drug exposure by taking into account clearance and markers of active disease as well as total drug delivered [21].

Interestingly, a larger percentage of the cases started with mPCDAI $\leq 7.5$ at the start of IFX induction infusions $(29.7 \%$ vs. $16.2 \%, \mathrm{P}=0.268$ ). This might be explained by the more recent data advocating a "top down" approach for which biologics and/or immunomodulators are used earlier in the course of disease, rather than starting with a 5-ASA. These results in pediatrics are conflicting, especially in pediatric CD $[1,22,23]$.

All patients initially enrolled in the study who developed high-level antibodies early on were switched to a different biologic (6 patients total, Fig. 1). The antibody levels ranged from 11-56 U/mL. For those final 37 who remained in the study and 34 of whom had 18-month data, only 3 patients developed low-level antibodies $(<10 \mathrm{U} / \mathrm{mL})$. This speaks to current evidence that dose escalation can be used to help clear low-level antibodies $(<10 \mathrm{U} / \mathrm{mL})$ [24].

Strengths of our study included matching with historic controls in a 1:1 fashion for mPCDAI at the start of infusions, age and sex. No major differences were noted between the cases and controls in terms of disease activity, therapy or demographics, so our results are likely to represent valid comparisons between 2 similar populations. 
Drug monitoring among cases was examined at set intervals, with trough levels from the same laboratory, so our results are likely to have high internal validity. The study took into account possible confounders that might change disease outcomes, including steroid exposure at the beginning of infusions, use of immunomodulators (even though the rates were similar between cases and controls) and the patient's health insurance.

Limitations to this study include the small sample size and the use of historic controls. Measurement of biomarkers, such as fecal calprotectin or lactoferrin, were cost-prohibitive and was not done for this pilot study. Another limitation is that dose intensification was left to the clinician's judgment and not controlled between the 2 cohorts. A longer follow up of these patients may further discern the value of TDM. In an attempt to evaluate patients over a longer period of at least 12-18 months, we did eliminate those patients from the study early on who were transitioned to another biologic for various reasons (lack of efficacy + high ADA levels), or moved to a new city/ transitioned to an adult provider etc., and for this reason, we did not use an intention-to-treat model but rather a complete case analysis. This does provide potential for bias and may have skewed our results to minimize the effect size of the intervention at hand. Unfortunately, as this was a prospective study assessing the intervention arm, we were not able to determine these missing data points. As this was a negative study, we did not pursue further statistical analysis such as imputation.

Lastly, since we used historic controls for the final analysis, we were not able to accurately assess the Paris or Montreal classification for comparison. We were able to calculate a validated mPCDAI scoring based on retrospective chart reviews that included ESR, albumin and disease activity.

In conclusion, all of our moderate-to-severe pediatric CD patients who received prospective TDM of IFX were in clinical remission at 18 months of follow up. This result was not statistically significantly different than the $88 \%$ clinical remission rate of the control group. The use of dose intensification strategies based on TDM appears promising in maintaining clinical remission, but careful clinician attention to patient symptoms and laboratory data fares similarly well. A collaborative multicenter study to yield a larger sample size and longer follow up may be helpful to tease out the observations of our pilot study.

\section{Acknowledgments}

We would like to thank the Prometheus Laboratory for analyzing our samples and Kim Shelly R.N. and all physicians in the Riley Pediatric Gastroenterology group for providing this project with their support and organization

\section{Summary Box}

\section{What is already known:}

- The role of therapeutic drug monitoring (TDM) of infliximab (IFX) for Crohn's disease (CD) is still controversial

- Higher anti-tumor necrosis factor drug levels are associated with better clinical outcomes and reduced drug immunogenicity

- Proactive TDM and dose adjustment to target serum levels are thought to optimize exposure of affected tissues to drug

\section{What the new findings are:}

- A wide variability in IFX trough levels is achieved following the 3-dose standard induction in children with $\mathrm{CD}$

- The trough IFX levels continued to increase over the follow-up period, probably because of greater dose intensification in this group

- Clinical remission is better in children who undergo proactive IFX TDM and dose optimization, although this approach was not statistically superior to careful clinical monitoring

- The children with proactive drug monitoring had more frequent dose intensifications

\section{References}

1. Tsui JJ, Huynh HQ. Is top-down therapy a more effective alternative to conventional step-up therapy for Crohn's disease? Ann Gastroenterol 2018;31:413-424.

2. Hyams J, Crandall W, Kugathasan S, et al; REACH Study Group. Induction and maintenance infliximab therapy for the treatment of moderate-to-severe Crohn's disease in children. Gastroenterology 2007;132:863-873; quiz 1165-1166.

3. Kugathasan S, Werlin SL, Martinez A, Rivera MT, Heikenen JB, Binion DG. Prolonged duration of response to infliximab in early but not late pediatric Crohn's disease. Am J Gastroenterol 2000;95:3189-3194.

4. Baldassano R, Braegger CP, Escher JC, et al. Infliximab (REMICADE) therapy in the treatment of pediatric Crohn's disease. Am J Gastroenterol 2003;98:833-838.

5. Colombel JF, Sandborn WJ, Reinisch W, et al; SONIC Study Group. Infliximab, azathioprine, or combination therapy for Crohn's disease. N Engl J Med 2010;362:1383-1395.

6. Feagan BG, McDonald JW, Panaccione R, et al. Methotrexate in combination with infliximab is no more effective than infliximab alone in patients with Crohn's disease. Gastroenterology 2014;146:681-688. 
7. Grossi V, Lerer T, Griffiths A, et al. Concomitant use of immunomodulators affects the durability of infliximab therapy in children with Crohn's disease. Clin Gastroenterol Hepatol 2015;13:1748-1756.

8. Kierkus J, Dadalski M, Szymanska E, et al. The impact of infliximab induction therapy on mucosal healing and clinical remission in Polish pediatric patients with moderate-to-severe Crohn's disease. Eur J Gastroenterol Hepatol 2012;24:495-500.

9. Bousvaros A. Mucosal healing in children with Crohn's disease: appropriate therapeutic goal or medical overkill? Inflamm Bowel Dis 2004;10:481-483.

10. Escher JC. Mucosal healing in pediatric Crohn's disease: pro/con balance. Inflamm Bowel Dis 2004;10:484.

11. Ruemmele FM, Veres G, Kolho KL, et al; European Society of Pediatric Gastroenterology, Hepatology and Nutrition. Consensus guidelines of ECCO/ESPGHAN on the medical management of pediatric Crohn's disease. J Crohns Colitis 2014;8:1179-1207.

12. Vande Casteele N, Ferrante M, Van Assche G, et al. Trough concentrations of infliximab guide dosing for patients with inflammatory bowel disease. Gastroenterology 2015;148:1320-1329.e3.

13. Carman N, Mack DR, Benchimol EI. Therapeutic drug monitoring in pediatric inflammatory bowel disease. Curr Gastroenterol Rep 2018;20:18.

14. Singh N, Rosenthal CJ, Melmed GY, et al. Early infliximab trough levels are associated with persistent remission in pediatric patients with inflammatory bowel disease. Inflamm Bowel Dis 2014;20:1708-1713.

15. Clarkston K, Tsai YT, Jackson K, Rosen MJ, Denson LA, Minar P. Development of infliximab target concentrations during induction in pediatric Crohn disease patients. J Pediatr Gastroenterol Nutr 2019;69:68-74.

16. Hyams J, Markowitz J, Otley A, et al; Pediatric Inflammatory Bowel Disease Collaborative Research Group. Evaluation of the pediatric
Crohn disease activity index: a prospective multicenter experience. J Pediatr Gastroenterol Nutr 2005;41:416-421.

17. Turner D, Griffiths AM, Walters TD, et al. Appraisal of the pediatric Crohn's disease activity index on four prospectively collected datasets: recommended cutoff values and clinimetric properties. Am J Gastroenterol 2010;105:2085-2092.

18. Ilowite MF, Al-Sayegh $\mathrm{H}, \mathrm{Ma} \mathrm{C}$, et al. The relationship between household income and patient-reported symptom distress and quality of life in children with advanced cancer: a report from the PediQUEST study. Cancer 2018;124:3934-3941.

19. Feuerstein JD, Nguyen GC, Kupfer SS, Falck-Ytter Y, Singh S; American Gastroenterological Association Institute Clinical Guidelines Committee. American Gastroenterological Association Institute Guideline on Therapeutic Drug Monitoring in Inflammatory Bowel Disease. Gastroenterology 2017; 153:827-834.

20. Minar P, Saeed SA, Afreen M, Kim MO, Denson LA. Practical use of infliximab concentration monitoring in pediatric Crohn disease. J Pediatr Gastroenterol Nutr 2016;62:715-722.

21. Bauman LE, Xiong Y, Mizuno T, et al. Improved population pharmacokinetic model for predicting optimized infliximab exposure in pediatric inflammatory bowel disease. Inflamm Bowel Dis 2020;26:429-439.

22. Punati J, Markowitz J, Lerer T, et al; Pediatric IBD Collaborative Research Group. Effect of early immunomodulator use in moderate to severe pediatric Crohn disease. Inflamm Bowel Dis 2008; 14:949-954.

23. Lee YS, Baek SH, Kim MJ, Lee YM, Lee Y, Choe YH. Efficacy of early infliximab treatment for pediatric Crohn's disease: a three-year follow-up. Pediatr Gastroenterol Hepatol Nutr 2012;15:243-249.

24. Cohen RZ, Schoen BT, Kugathasan S, Sauer CG. Management of anti-drug antibodies to biologic medications in children with inflammatory bowel disease. J Pediatr Gastroenterol Nutr 2019;69:551-556. 


\section{Supplementary material}

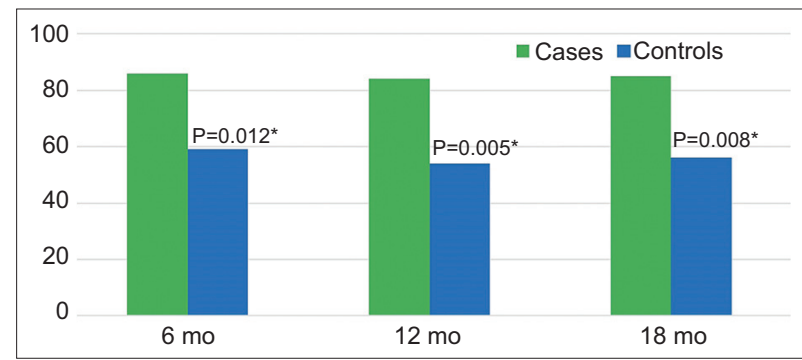

Supplementary Figure 1 Percentage of IFX dose $>5 \mathrm{mg} / \mathrm{kg}$ at 6,12 and 18 months

*Significant at a P-value $<0.05$. Values are frequencies (percentages) with P-values from McNemar's tests

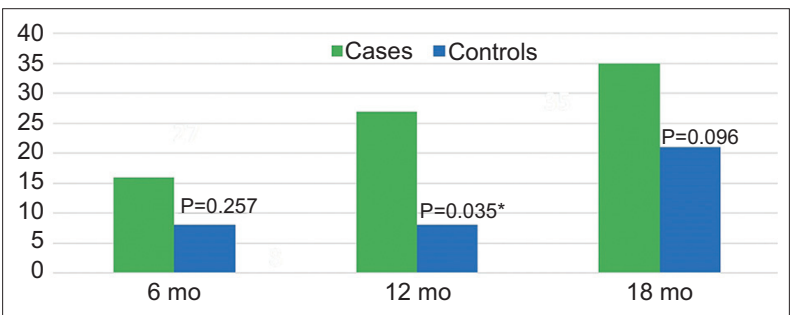

Supplementary Figure 2 Percentage receiving IFX infusion $<8$ week intervals at 6,12 and 18 months

${ }^{*}$ Significant at a P-value $<0.05$. Values are frequencies (percentages) with P-values from McNemar's tests 